\title{
Weather monitoring and forecasting system using IoT
}

\author{
Balakrishnan Sivakumar ${ }^{1,{ }^{*}}$ and Chikkamadaiah Nanjundaswamy ${ }^{2}$ \\ 1 Department of Telecommunication Engineering, Dr. Ambedkar Institute of Technology, Bangalore, Karnataka, India. \\ 2 Department of Civil Engineering, Dr. Ambedkar Institute of Technology, Bangalore, Karnataka, India.
}

Global Journal of Engineering and Technology Advances, 2021, 08(02), 008-016

Publication history: Received on 21 June 2021; revised on 01 August 2021; accepted on 03 August 2021

Article DOI: https://doi.org/10.30574/gjeta.2021.8.2.0109

\begin{abstract}
The system proposed is an advanced solution for weather monitoring that uses IoT to make its real time data easily accessible over a very wide range. The system deals with monitoring weather and climate changes like temperature, humidity, wind speed, moisture, light intensity, UV radiation and even carbon monoxide levels in the air; using multiple sensors. These sensors send the data to the web page and the sensor data is plotted as graphical statistics. The data uploaded to the web page can easily be accessible from anywhere in the world. The data gathered in these web pages can also be used for future references. The project even consists of an app that sends notifications as an effective alert system to warn people about sudden and drastic weather changes. For predicting more complex weather forecast that can't be done by sensors alone we use an API that analyses the data collected by the sensors and predicts an accurate outcome. This API can be used to access the data anywhere and at any time with relative ease and can also be used to store data for future use. Due to the compact design and fewer moving parts this design requires less maintenance. The components in this project don't consume much power and can even be powered by solar panels. Compared to other devices that are available in the market the Smart weather monitoring system is cheaper and cost effective. This project can be of great use to meteorological departments, weather stations, aviation and marine industries and even the agricultural industry.
\end{abstract}

Keywords: API; IoT; Monitoring; Weather; Forecasting; Sensor

\section{Introduction}

Present day innovations in technology mainly focus on controlling and monitoring of different devices over wirelessly over the internet such that the internet acts as a medium for communication between all the devices. Most of this technology is focused on efficient monitoring and controlling of different. An efficient environmental monitoring system is required to monitor and assess the weather conditions in case of exceeding the prescribed level of parameters (e.g., noise, CO and radiation levels) and for gathering data for research purposes (amount of rainfall, windspeed etc.). A system is considered as a smart system when the device equipped with sensors, microcontrollers and various software applications becomes a self-protecting and self-monitoring system.

The recent changes in climate have increased the importance of environment monitoring. The current advance in the field of technology and economy are hardly a significant impact over the environment, have led to serious concern regarding pollution and climate change. The intergovernmental panel on climate change (IPCC) in the report confirms that human activities are having an unequivocal and continuously increasing influence on the climate system, with recent changes that are unprecedented over decades of millennia.

\footnotetext{
${ }^{*}$ Corresponding author: Balakrishnan Sivakumar

Department of Telecommunication Engineering, Dr. Ambedkar Institute of Technology, Bangalore, Karnataka, India. 
In this contest, environmental monitoring represents a fundamentals instrument for gathering relevant information about the ecosystem, leading to new knowledge and understanding and for ultimately adaptation and mitigation actions that address the degradation of the biosphere.

Event Detection based and Spatial Process Estimation are the two categories to which applications are classified. Initially the sensor devices are deployed in environment to detect the parameters (e.g., Temperature, Humidity, Pressure, LDR, noise, CO and radiation levels etc.) while the data acquisition, computation and controlling action (e.g., the variations in the noise and CO levels with respect to the quantified levels). Sensor devices are positioned at different locations to collect the data to forecast the behavior of a particular area of interest. The main aim of this paper is to design and implement a resourceful monitoring system through which the required parameters are monitored remotely using internet and the data gathered from the devices are stored in the cloud and to project the predictable trend on the web browser.

A solution for monitoring temperature and CO levels i.e., any parameter value crossing its threshold value ranges, for example CO levels in air in a particular area exceedingly the normal levels etc., in the atmosphere using wireless embedded computing system is proposed in this paper. The solution also provides an intelligent remote monitoring for a particular area of interest. In this paper we also current results of collected or sensed data with respect to the normal or specified ranges of particular parameters. The embedded system is an integration of sensor devices, wireless communication which enables the user to remotely access the various parameters and store the data in cloud.

\section{Literature review}

In today's world many pollution monitoring systems are designed by different environmental parameters. Existing system model is presented IOT based Weather monitoring and reporting system where you can collect, process, analyses, and present your measured data on web server. Wireless sensor network management model consists of and device, router, gateway node and management monitoring center. End device is responsible for collecting wireless sensor network data, and sending them to parent node, the data are sent to gateway node from parent node directly or by router. After receiving the data from wireless sensor network, gateway node extracts data after analysing and packaging them into Ethernet format data, sends them to the server. Less formally, any device that runs server software could be considered a server as well. Servers are used to manage network resources. The services or information provided through the Internet that are connected through LAN and made available for users via smart phones, web browser or other web browser devices to make the system more intelligent, adaptable and efficient.

\section{Existing system}

The existing weather monitoring systems generally use weather stations that use multiple instruments such as thermometers, barometers, wind vanes, rain gauge etc. to measure weather and climate changes. Most of these instruments use simple analog technology which is later physically recorded and stored in a data base. This information is later sent to news reporting stations and radio stations where the weather report [1] is given.

\subsection{Limitations of the existing system}

- Existing weather monitoring systems that are used in the field generally consist of unconventional and heavy machinery that consists of numerous moving parts that require constant maintenance and need to be manually monitored and changed frequently.

- Power requirements are one of many major constraints as these instruments are generally sited far from main power supply. This adds to the cost of using such instruments.

- The use of thermometers to measure external temperature; however accurate is still outdated and constantly needs to be manually checked for any change in temperature.

- Data that is collected by the instruments needs to be manually transferred from the logger to a laptop or computer via a cable.

- Existing systems consist of large and heavy instruments that occupy a lot of space hence making it difficult to install them in remote location and places which have limited space.

- The instruments used in the existing systems are expensive and add up to the already high cost of installation and maintenance. 
- The current system always faces problems such as delay in warning people about bad weather and sudden changes in the forecast.

\section{Proposed system}

The system proposed is an advanced solution for weather monitoring that uses IoT to make its real time data easily accessible over a very wide range. The system deals with monitoring weather [1] and climate changes like

- Temperature, humidity by using the DHT11 sensor

- Wind speed using an Anemometer

- Light intensity using an LDR

- UV radiation using a GY8511 solar sensor

- Carbon monoxide levels in the air using MQ7

- Soil moisture using Hygrometer

- Ultrasonic sensor for rain water level,

- Raindrop sensor for detecting rainfall or snow fall.

\section{Feature and advantages of the proposed system}

- Our proposed 'Smart weather monitoring system' unlike conventional weather monitoring instruments is very small and compact allowing it to be installed easily on rooftops.

- It is light and portable; this advantage allows us to easily carry it to remote location for installation. Due to its design, it can be easily be carried by a weather balloon to measure atmospheric changes at high altitudes.

- The power requirements for our system (sensors and boards) is much less compared to the existing instruments in the market hence enabling us to use solar cells as power supply [2]. This not only cuts down on cost but allows us to leave the monitoring system in remote, areas where power is not easily available, for long periods of time. Addition of solar panels also helps our design be eco-friendly.

- The sensors used in our product are much cheaper compared to the ones that are used in the existing weather monitoring systems making our design more cost effective.

- These sensors send the data to a web page and the sensor data is plotted as graphical statistics. The data uploaded to the web page can easily be accessible from anywhere in the world. The data gathered in these web pages can also be used for future references [3]. Unlike the existing system where data has to be physically transferred.

- Due to the presence of fewer moving parts less amount of maintenance will be needed cutting down on maintenance charges.

- The product even consists of an app that sends notifications as an effective alert system to warn people about sudden and drastic weather changes. This works as an efficient warning system for bad weather and storms.

- For predicting more complex weather forecast that can't be done by sensors alone we use an API with the help of a Raspberry pi that analyses the data collected by the sensors and predicts an accurate outcome. This API can be used to access the data anywhere and at any time with relative ease and can also be used to store data for future use.

\section{6. system architecture}

The implemented system consists of an Arduino Uno which is used as a main processing unit for the entire system and all the sensor and devices can be connected with the microcontroller. The sensors can be operated by the microcontroller to retrieve the data from them and it processes the analysis with the sensor data [3]. The processed data can be uploaded and stored in a website to function as a data base using node MCU and Ubi dots. 


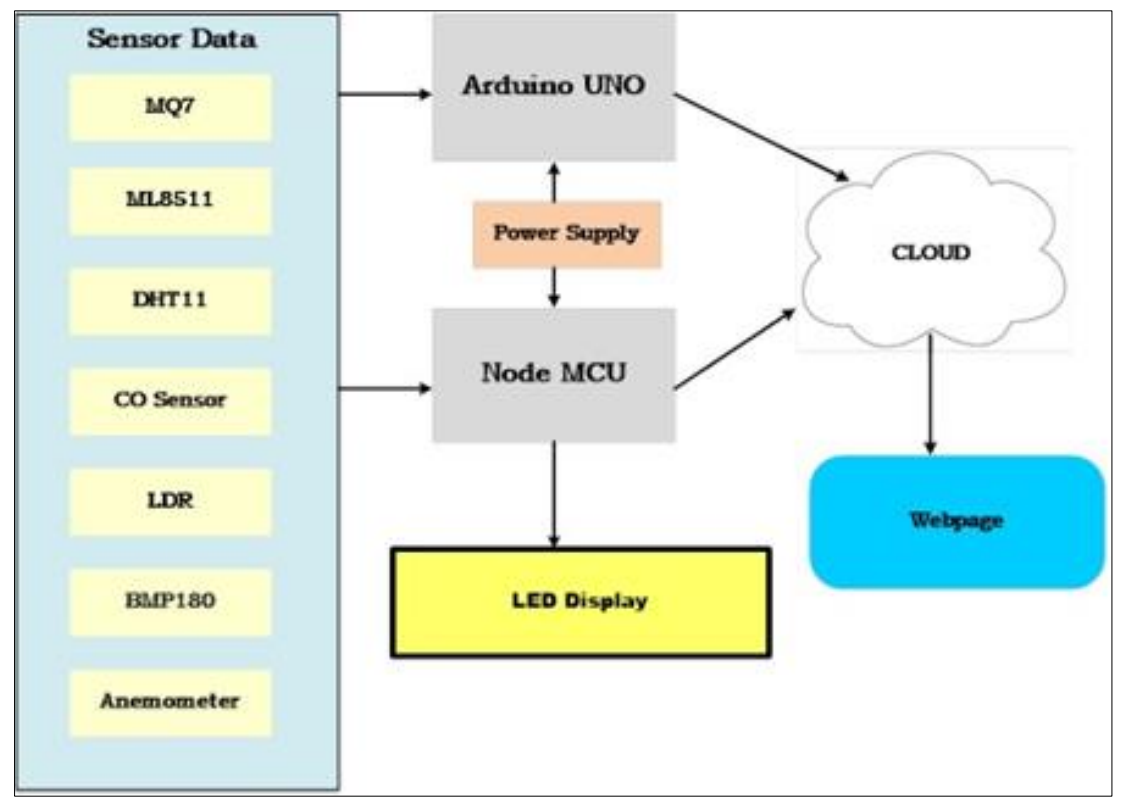

Figure 1 Block diagram of Implementation

\section{Hardware requirement}

- Arduino UNO

- Node MCU

- LDR-Light Dependent Resistor

- CO Sensor- Carbon Monoxide (CO) sensor

- DHT11-Temperature and Humidity Sensor

- Anemometer- to determining the speed of wind.

- ML8511- ultraviolet light sensor

- BMP180- Pressure Sensor

- Hygrometer.

- Raindrop sensor for detecting rainfall or snow fall.

\subsection{Middleware Language}

C, C++, Python, Mosquitto broker software

\subsection{Servers with root access}

- VPS or Dedicated cloud server

- Work station (local)

- Dedicated PCs

\subsection{Frontend development}

HTML, CSS, React Js

\subsection{Backend development}

Node JS and Express Js

\subsection{Database}

Nosql or Mongo DB 


\section{Software requirement}

- Dark Sky.net- Dark Sky is an open source Internet of Things (IOT)application and API to store and retrieve data from things using the HTTP protocol over the Internet or via a Local Area Network[4]. We interface it with the help of a Raspberry pi.

- Android App-The main purpose of this app is to provide notifications on weather updates and to act as warning system in case of bad weather. The app will obtain

- the necessary information through the existing database.

\section{Implementation}

The proposed system can be implemented in a 4- tier model with the functions of everyone modules developed for monitoring the different weather parameters. The tier 1 is the environment, sensor devices in tier 2, sensor data acquisition and decision making in tier 3 and warning notification in tier 4 . Here, the tier 1 provides information about the parameters under the region which is to be monitored. Tier 2 deals with the sensor devices with suitable characteristics, features and each of these sensor devices are operated and controlled based on their sensitivity as well as the range of sensing. In between tier 2 and tier 3 necessary sensing and controlling actions will be taken depending upon the conditions, like fixing the threshold value, periodicity of sensing, messages etc. Based on the data analysis performed in between tier 2and tier 3 and also from previous experiences the parameter threshold values during critical situations or normal working conditions are determined [6].

Tier 3 describes about the data acquisition from sensor devices and also includes the decision making. Which specify the condition the data is representing which parameter?

In the proposed model tier 4 deals with the intelligent environment. Which means it will identify the variations in the sensor data and fix the threshold value depending on the identified levels [5]. In this tier sensed data will be processed, stored in the cloud and accordingly the notification will be sent.

Based on the framework we have identified a suitable implementation model that consists of different sensor devices and other modules. In this implementation model we use a Node MCU for sensing and storing the data in cloud. Inbuilt ADC and Wi-Fi module attaches the embedded device to internet. Sensors are connected to Node MCU board for monitoring, ADC will convert the corresponding sensor reading to its digital value and from that value the corresponding environmental parameter will be evaluated.

\subsection{Algorithm for checking the sim 300 using PC}

- Insert a SIM card on the board into the SIM tray. Attach the board to the computer's USB port using a RS232 to USB serial cable.

- To read the text being sent by the modem windows has a built-in serial monitoring software called Hyper terminal.

- Find it at Start ->> Programs - >>Accessories ->> Communications ->>Hyper terminal

- Enter connection name.

- Select the serial port at which the modem is connected under the "Connect Using "option.

- Select Baud rate 9600(default) and Flow control none. The GSM module works on a serial communication as well as TTL interface that can work within a range of speeds from $1200 \mathrm{bps}$ to $1152000 \mathrm{bps}$.

- Enter "AT" in the Hyper terminal, the board will respond "OK" if all the things are connected correctly.

- The yellow LED is used to display the network status.

- LED Off means SIM300 is not receiving signal.

- $64 \mathrm{~ms}$ to 0.8 Sec Off means SIM300 is having weak or no network.

- $64 \mathrm{~ms}$ to 3 Sec Off means SIM300 found network.

\subsection{Applications, Advantages and Future enhancements of IOT weather monitoring system}

- IOT weather reporting system has an application to farmers as well. The weather forecasting plays a very important role in the field of agriculture [7]. 
- IOT weather monitoring project proves helpful for monitoring weather at places like a volcano, rain forests. It is quite difficult for a human being to stay for a longer time at such places. Or even areas that are exposed to radioactive leakage.

- IOT weather monitoring system project using IoT supporting controller is fully automated. It does not require any human attention.

- You can get a prior alert of the weather conditions. Suppose you are planning to visit a place and you want to know the weather parameters over that place, then you can just visit a website IoT portal.

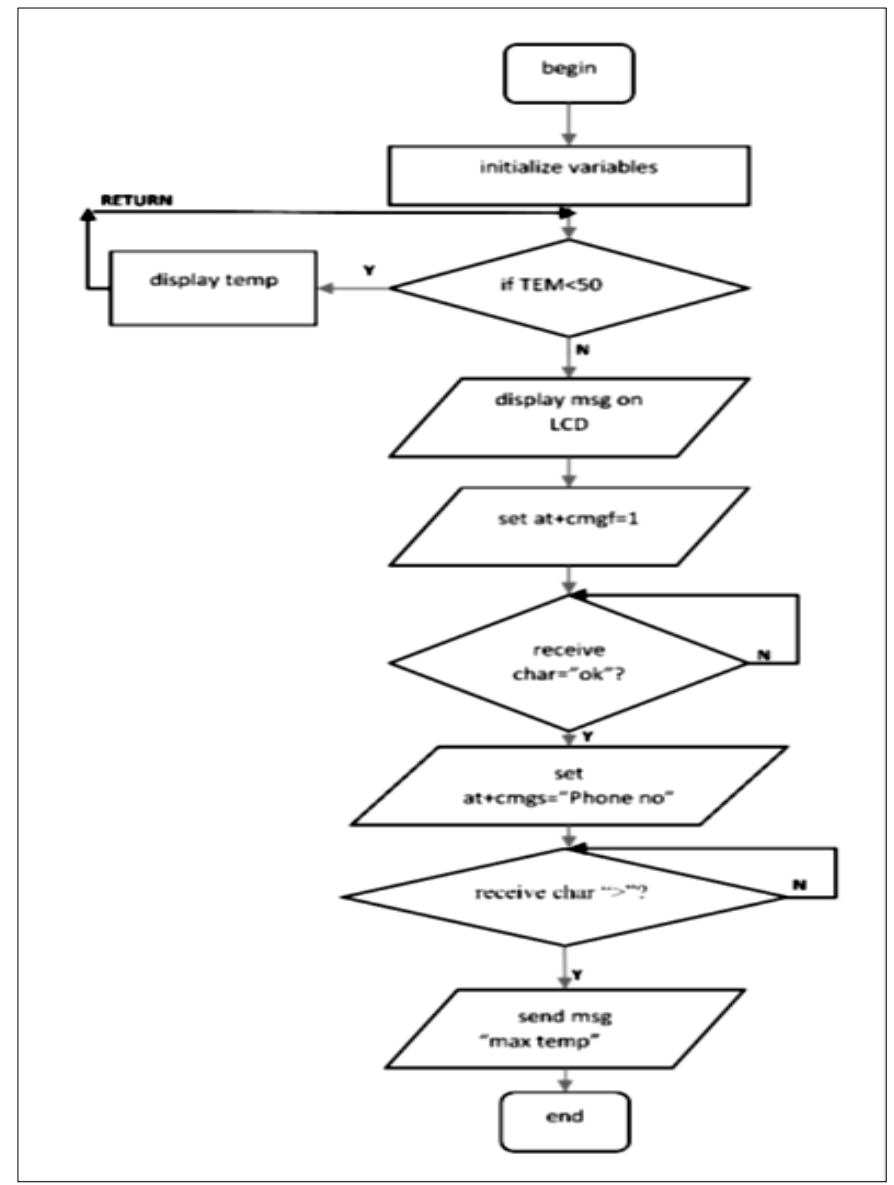

Figure 2 Flowchart for sending Message

\subsection{Key areas that are benefited with IoT weather forecasting technology}

As mentioned in the above, accurate weather report is forecasted directly or indirectly influences other sectors of economy to a great extent, it will further raise the need of a system that facilitates higher accuracy of real time monitoring and future weather prediction. Below you can have a look at key sectors that are benefited with IoT weather forecasting technology.

Agriculture - Agricultural process i.e. preparation of soil, sowing, irrigation, harvesting and storage of crops is directly dependent on weather condition leaving farmers vulnerable to weather hazards [5].

Development and expansion of IoT technology for weather forecasting will deliver vital weather prediction to farmers and accordingly farmers may use the intelligence to improve their crop fertility and cost along with taking essential steps to diversify weather hazards. Timely and accurate delivery of weather forecast will ensure higher productivity and lower the risk of weather hazard. 


\section{Result}

The second part of the GSM port and the SMS service is done by either web gateway interface or the GSM modem. The SMS regarding the current weather statistics will be displayed on the user's/receiver's cell phone. The weather to be forecasted based on the current and previous data will generate the expected result as shown in Figure 3.

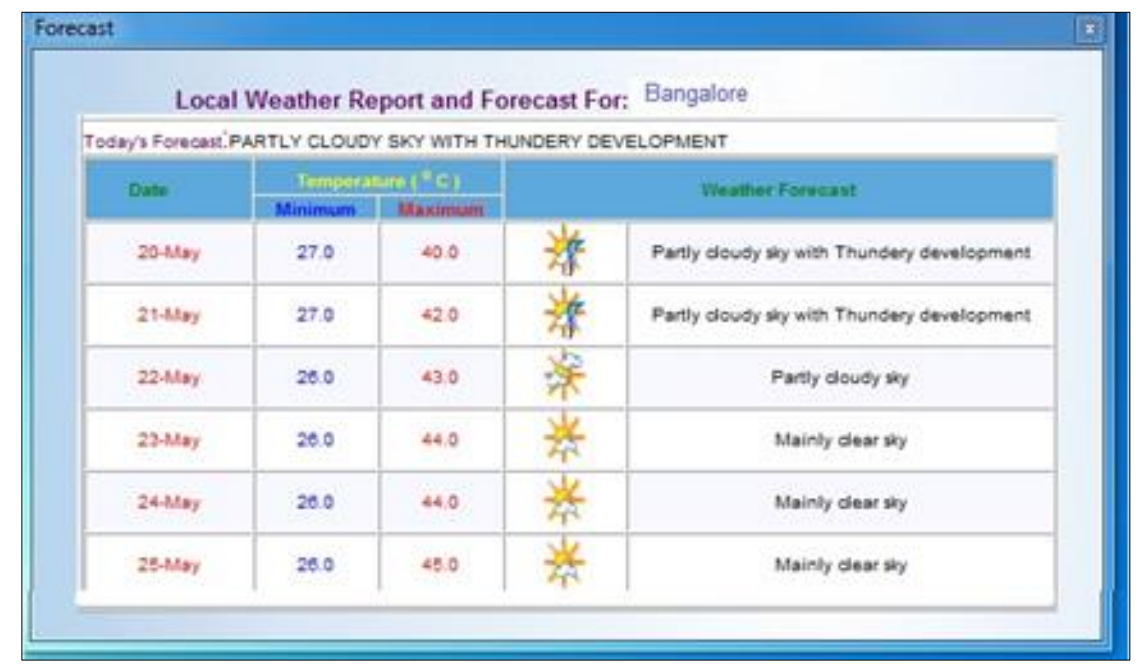

Figure 3 Weather forecast report

Wireless weather monitoring system is a microcontroller-based project and can be implemented practically as displayed. In this project the temperature sensor (LM 35) is connected to ATMEGA32 microcontroller and temperature result is sent to GSM modem which is simultaneously displayed on LCD. Based upon the experimentation and implementation, we came to observe that the temperature can be monitored in an isolated place using LM35 (temperature sensors) and can be sent via SMS to a distant users mobile [10].

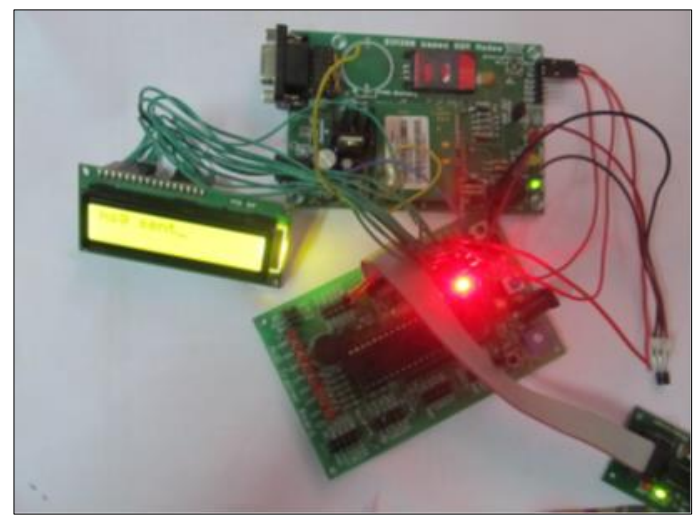

Figure 4 Working model of Wireless Weather Monitoring System

\section{Conclusion \& Discussions}

To implement this need to deploy the sensor devices in the environment for collecting the data and analysis. By deploying sensor devices in the environment, it will record real time data. It can cooperate with other objects through the network. Then the collected data and analysis results will be available to the end user through the Wi-Fi. The smart way to monitor environment and an efficient, low-cost entrenched system is presented with different models in this paper.

Weather station is a form of application of science and technology to know and predict the weather condition at a particular body. The weather station is designed to collect quantitative data about the weather condition of a region in order to be able to known and predict weather conditions in a region [8]. Human activities depend on whether factors. 
Weather is an air condition that lasts for a short period of time so the short-term weather forecasting process should be done as quickly and accurately as possible.

In the proposed architecture purposes of different modules were discussed [9]. The noise and air pollution monitoring system with Internet of Things (IoT) concept experimentally tested for monitoring two parameters. It also sent the sensor parameters to the cloud (Google Spread Sheets). This data will be cooperative for future analysis and it can be easily shared to other end users. This model can be further expanded to monitor the developing cities and manufacturing zones for pollution monitoring. To protect the public health from pollution, this model provides an efficient and low-cost solution for unceasing monitoring of environment.

Additions that can be made to improve the system

- $\quad$ Powering the device using solar panels.

- Suspending the device from a weather balloon so that it can be used to record atmospheric parameters at high altitudes and remote and inaccessible areas.

- Use of a though exterior cover for the system that will act as a protective cover enabling the device to function in harsh weather conditions.

- Designing a method to mount the weather monitoring device onto a buoyant platform like a buoy hence enabling the system to measure weather changes over the sea. This data can also be shared to Cargo ships and other nautical industries conducting operations within the area.

- Using silica gel to prevent condensation on the exterior cover as condensation might affect the sensors readings.

\section{Compliance with ethical standards}

\section{Acknowledgments}

I wish to acknowledge the support given by Research Centre, Dr. Ambedkar Institute of Technology, Banaglore, Karnataka, India.

\section{Disclosure of conflict of interest}

There are no conflicts of interest in connection with this paper, and the material described is not under publication or consideration for publication elsewhere.

\section{References}

[1] International Journal of Trend in Scientific Research and Development (IJTSRD)Volume: 3 | Issue: 3 | Mar-Apr 2019 Available Online: www.ijtsrd.com e-ISSN: 2456 - 6470 @ IJTSRD | Unique Paper ID - IJTSRD21677 | Volume - 3 | Issue - 3 | Mar-Apr 2019 Page: 365"IOT Based Weather Monitoring and Reporting System Project "Anita M. Bhagat, Ashwini G. Thakare, et al.

[2] Internet of Things (IOT) Based Weather Monitoring system .Bulipe Srinivas Rao, Prof. Dr. K. Srinivasa Rao, Mr. N. Ome International Journal of Advanced Research in Computer and Communication Engineering ISO 3297:2007 Certified.September 2016; 5(9).

[3] Keshav kumar singh, S Styline Chirmaxo. Design of Wireless Weather Monitoring System, Department of Electronics \& Communication Engineering National Institute of Technology Rourkela. 2013.

[4] Pranjali Wankhede et al. A Review on Weather Forecasting Systems Using Different Techniques and Web Alerts, International Journal of Advanced Research in Computer Science and Software Engineering. February - 2014; 4(2): 357-359.

[5] GV Satyanarayana, Sd Azharuddin. Wireless Sensor Based Remote Monitoring System for Agriculture Using ZigBee and GPS, Conference on Advances in Communication and Control Systems (C) 2013.

[6] Baste, P and Dighe, D “ Low Cost Weather Monitoring Station Using Raspberry PI”, International Research Journal of Engineering and Technology (IRJET) Vol. 4, Issue 05, May 2017. 
[7] Iswanto and Muhammad, H."Weather Montoring System with Remote Radio Frequency Wireless Communication", International Journal of Embedded System and Application (IJESA), Vol. 2 No. 3 September 2012.

[8] Patil, M., Pachpande, S.R., Chaudari, JP., and Rane, K.P., "Study of Literature on Weather Monitoring System" International Journal of Computer Application, Vol. 153, No. 3 November 2016.

[9] Soe, L, Lwin, K and Tun, H, Implementation of Microcontroller Based Sensing Unit in Transmitter for Wireless Weather Station, International Journal of Science, Engineering and Technology Research (IJSETR), Volume 3, Issue 6, June 2014.

[10] https://circuitdigest.com/microcontroller-projects/esp12-nodemcu-based-iot-weather-station.

\section{Author's short biography}

Dr. B. Sivakumar has got the academic qualification as B.E., M.E., PGDBA., Ph.D. Presently as
Professor Dept. of ETE (Govt. Aided), and graduated from Madurai Kamaraj University in the
field of Electronics and Communication. Also obtained his Master degree from PSG College of
Technology, Bharathiar University in the field of Applied Electronics. Has been awarded
Doctoral Degree in the field of Information \& Communication Engineering from Anna
University, Chennai. Has got a rich teaching experience of 31 years. Has to his credit 44
International/National in peer-reviewed Journals and 120 Conference Papers. He has already
guided 3 Ph.D Scholars and presently guiding 5. He has filed 2 International patent to his credit.
He has completed 2 AICTE Research grant projects to the tune of 20 lakhs. Presently carrying
out 3 AICTE- AQIS programs. He is an Editor /Reviewer for 4 International Journals.

